\title{
Reconstructed State Space Features for Classification of ECG Signals
}

\author{
Soheil Pashoutan ${ }^{1 * \odot}$, Shahriar Baradaran Shokouhi ${ }^{2}$
}

\begin{abstract}
Background: Cardiac arrhythmias are considered as one of the most serious health conditions; therefore, accurate and quick diagnosis of these conditions is highly paramount for the electrocardiogram (ECG) signals. Moreover, are rather difficult for the cardiologists to diagnose with unaided eyes due to a close similarity of these signals in the time domain.

Objective: In this paper, an image-based and machine learning method were presented in order to investigate the differences between the three cardiac arrhythmias of VF, VT, SVT and the normal signal.

Material and Methods: In this simulation study, the ECG data used are collected from 3 databases, including Boston Beth University Arrhythmias Center, Creighton University, and MIT-BIH. The proposed algorithm was implemented using MATLAB R2015a software and its simulation. At first, the signal is transmitted to the state space using an optimal time delay. Then, the optimal delay values are obtained using the particle swarm optimization algorithm and normalized mutual information criterion. Furthermore, the result is considered as a binary image. Then, 19 features are extracted from the image and the results are presented in the multilayer perceptron neural network for the purpose of training and testing.
\end{abstract}

Results: In order to classify N-VF, VT-SVT, N-SVT, VF-VT, VT-N-VF, N-SVTVF, VT-VF-SVT and VT-VF-SVT-N in the conducted experiments, the accuracy rates were determined at $99.5 \%, 100 \%, 94.98 \%, 100 \%, 100 \%, 100 \%, 99.5 \%, 96.5 \%$ and $95 \%$, respectively.

Conclusion: In this paper, a new approach was developed to classify the abnormal signals obtained from an ECG such as VT, VF, and SVT compared to a normal signal. Compared to Other related studies, our proposed system significantly performed better.

Citation: Pashoutan S, Baradaran Shokouhi Sh. Reconstructed State Space Features for Classification of ECG Signals. J Biomed Phys Eng. 2021;11(4):535-550. doi: 10.31661/jbpe.v0i0.1112.

\section{Keywords}

Ventricular Fibrillation; Tachycardia; Ventricular; Neural Networks; Computer

\section{Introduction}

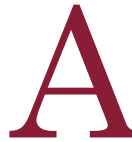

$n$ electrocardiogram (ECG) is a display of the electrical activities of a heart. An electrocardiogram records the signals using electrodes placed over the patient's body and the results essentially indicate the origin and diffusion of electrical potential in the heart muscle [1]. This can be regarded as a non-invasive, safe, and quick method to illustrate the cardiac arrhythmias in the time domain, as well as diseases affecting the cardiovascular system [2]. Any irregularities

${ }^{1} \mathrm{MSc}$, Department of

Electrical, Iran University

of Science and Technol-

ogy, Tehran, Iran

${ }^{2} \mathrm{PhD}$, Department of

Electrical, Iran University

of Science and Technol-

ogy, Tehran, Iran

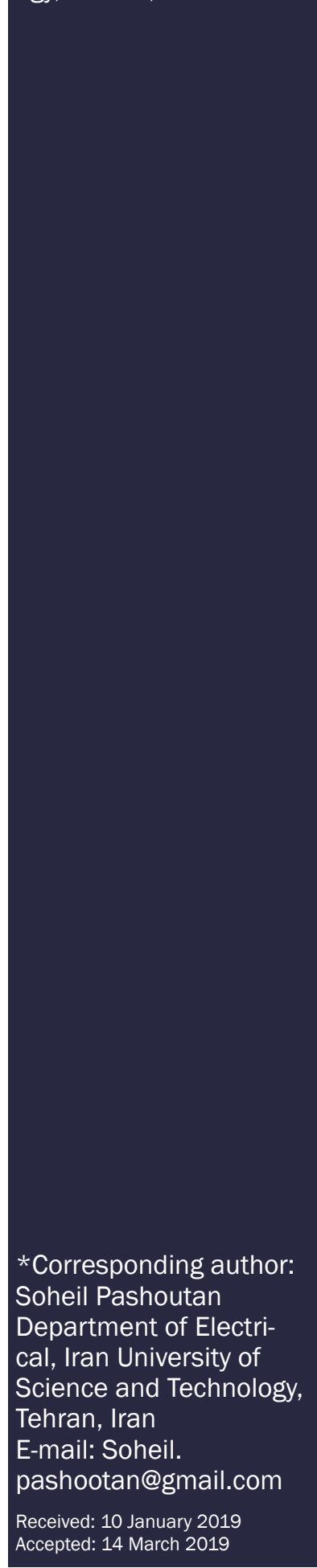


in the heart rate, pace, and transmission of electrical pulses, in the heart, can be considered as a type of cardiac arrhythmia, where cardiologists can detect the type of disease based on the results obtained from an electrocardiogram. Moreover, various symptoms can cause heart disease; however, atherosclerosis and hypertension are two of the most common causes [3]. Additionally, the physiology and morphology of the body change with aging, which leads to alterations in the cardiovascular system performance, and consequently, the risk of developing cardiovascular disease increases [4]. Different arrhythmias exist, including Atrial Fibrillation (AF), Ventricular Tachycardia (VT), Ventricular Fibrillation (VF), Left Bundle Branch Block (LBBB), Right Bundle Branch Block (RBBB), Left Anterior Hemiblock (LAHB), Atrial Premature Complex (APC), Premature Ventricular Complex (PVC), and Supraventricular Tachycardia (SVT) [5]. Furthermore, none of every arrhythmia detected by ECG is dangerous. As a rule, due to the crucial effect of ventricles on pumping blood, the arrhythmias involving the ventricle are more dangerous [6]. According to several statistical studies, about $80 \%$ of the Sudden Cardiac Deaths (SCD) are the result of automatic Supraventricular Tachyarrhythmia (SVTA), VT, and VF. Therefore, it is rather imperative to diagnose these types of arrhythmias, particularly for patients who have an implanted defibrillator in their bodies. In order to prevent loss of life, appropriate treatment measures should be taken within a few minutes, once these types of arrhythmias are diagnosed [7-9]. Thus, in this paper, a method was proposed for separate classifying of SVT, VF, VT arrhythmias and the normal signal. The classification of different heart rates can be regarded as one of the most important steps in determining the type of arrhythmia. However, since the classification of different heart rates is extremely difficult and rather time-consuming in a number of cases, any automated processing sample, which can be used to facilitate the classification of heart rates, has been considered in various studies.

Cardiac signals have relatively similar appearances in terms of time domain; however, their nature widely varies. Over the past few decades, a few algorithms have been proposed to distinguish different types of arrhythmias [10-23]. These studies are divided according to the extracted features, the classification method, data, and the training and testing strategy. In addition, these algorithms include methods based on ECG signals processing in the time domain such as using the passing through threshold criterion [10], the autocorrelation function [11], the conversion of a signal into a binary signal and evaluating complexity [12]. Algorithms in the frequency domain have been proposed in other articles such as the band-stop filter in order to estimate the leakage rate and the spectral analysis method [13]. In a number of conducted studies, new approaches were employed for the detection of VF arrhythmia, including wavelet transform [14], Support Vector Machine (SVM) [15], and an algorithm for classifying VF arrhythmia using a learning machine method [8]. Finally, other articles have been published for the classification of arrhythmias. Where several features of the ECG signal were extracted, including spectral features, entropy approximation, direct detection of the feature [8] and time-frequency analysis [16] in neural networks. Moreover, a number of systems, proposed to classify ECG signals, have been evaluated in the literature [17-21].

Amann et al. [22] transmitted the VT signals to the state space in order to classify them and their normal signals, where the result was obtained as an image of several pixels and VT classification was performed by counting the number of pixels. Furthermore, this was achieved by considering the signal's sample as the state variable $\mathrm{X}(\mathrm{t})$, which is shown with respect to $X(t+\tau)$ where the classification was obtained by pixel counting. Sarvestani et al. [23] added effective masks to the obtained 
image in the state space in order to increase the accuracy of VT and VF signal classification, where classification was performed in this new condition. However, these studies were only able to classify a limited portion of signal classes, in several specific articles [22, 23], only two data classes were classified.

Despite the fact that various automatic heart rate classification methods have been investigated in previously conducted studies, it is hardly feasible to compare the effectiveness of these methods since different data and features were used in each of these methods.

Moreover, the techniques used for the classification of signals, via the signal power spectrum, may not have identical resolution capacity; however, the reconstruction of heart rate signals in the state space results in a highly desirable resolution. Additionally, the established theories of the state space ensure that each system dynamics can be described by the reconstruction of the state space of each state variable, if the state space of the reconstructed state is larger than twice the cell count in terms of dimension.

In this paper, the state-space reconstruction method was used in order to achieve a more comprehensive and appropriate space, which is less dependent on the individual patient. Moreover, this method was employed in order to obtain a higher degree of classification, and consequently, to create a desirable training set. This method is able to generate an automatic heart rate classification, which is not only independent of the individual patient but also highly efficient in that regard. In this study, the normalized mutual information criterion between two images and an optimization algorithm was employed in order to determine $\tau$ and to obtain best values, respectively. Furthermore, the concept of masks, used in previous studies [23], was employed in order to develop highly effective masks to extract six features. Image processing methods were utilized to extract six more features that the final seven extracted features were parts of a tex- ture features, extracting information associated with the relative positions of the image pixels in relation to each other. 19 features were considered for the optimal classification and as training validation and testing data for the neural network.

\section{Material and Methods}

\section{Cardiac Arrhythmias}

In this simulation study, arrhythmia is referred to a group of heart signals when an abnormal condition exists in the heart's electrical activity and rhythm [5]. While several arrhythmias, such as VT, VF, and SVT are extremely dangerous and considered to be lifethreatening, other arrhythmias may not pose a significant threat. Ventricular fibrillation is a type of cardiac arrhythmias, where electrical stimulation deviates from following a specific pathway in the heart's electrical conduction system. If VF occurs, various points in the ventricles begin to send rapid impulses, resulting in a highly irregular depolarization and desynchronization of the ventricles, in other words, the heart muscle quivers instead of effective contraction and pumping. Therefore, pumping essentially stops, then the levels of cardiac outlet and effective blood flow rapidly and significantly reduce and eventually, death occurs during a few minutes due to cardiac arrest [24]. Ventricular tachycardia is another type of arrhythmias, in which the heart rate increases to more than $100 \mathrm{bpm}$. This rise in the heart rate may sometimes have a physiological origin and occur at the time when the body needs higher levels of oxygen (such as exercise). However, this can considerably elevate (250 bpm) in an individual suffering from VT, causing interference in pumping and dizziness and cardiac arrest. VT may also develop into VF without any significant sign, which is a deadly disease [5]. Supraventricular tachycardia is a different type of arrhythmias, which is not as dangerous as the previously described arrhythmias; however, it should be carefully 
identified and recognized by the cardiologist in order to provide the appropriate treatment and therapies [7]. In order to show the existing similarities, SVT, VF, VT, and normal signals are shown in Figure 1.

\section{Database}

The data used in this article were extracted from a review of more than 500 signal samples obtained from four databases [25]. They include Creighton University Ventricular Tachyarrhythmia Database (CUDB), Normal Sinus Rhythm Database (NSRDB) from Boston Beth University Arrhythmias Center, and finally, Ventricular Fibrillation Database (VFDB) and Supraventricular Arrhythmia Database (SVDB) from the MIT-BIH University. 121 signal samples with a length of 1 minute were obtained, which 40, 40, 20, 21 of them were VF, normal, VT, and SVTA signal samples, respectively. The frequency of signals associated with the CUDB and VFDB was determined at $250 \mathrm{~Hz}$ and also $128 \mathrm{~Hz}$ for The NSRDB and SVDB. In this study, the proposed system provides an opportunity to compare all of the data; as a result, all of the extracted data from different databases should possess a matched frequency before any further analysis. In this simulation study, the proposed algorithm is simulated using MATLAB R2015a, and the laptop used in this study had the following configurations: 32-bit windows 7, Intel Core i7 $2.53 \mathrm{GHz}, 4$ GB RAM.

\section{Method}

The block diagram of the proposed algo-

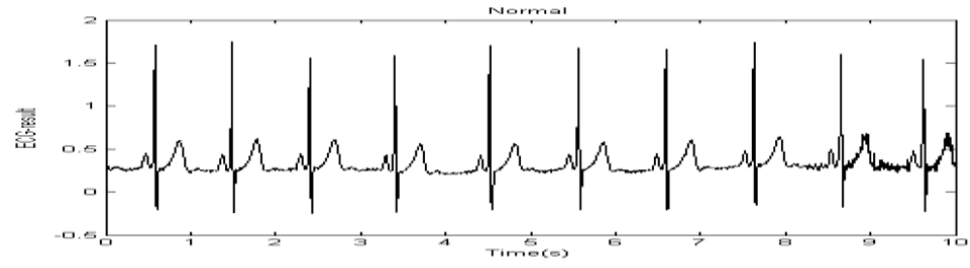

A

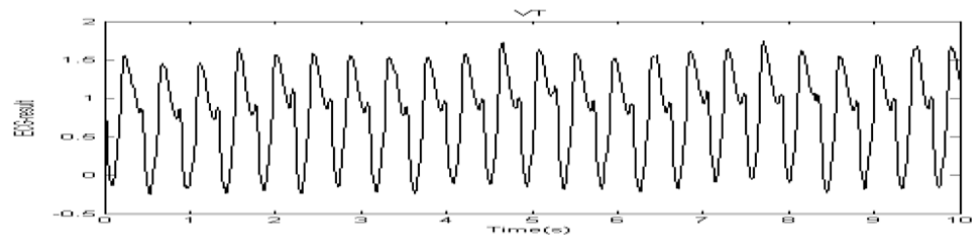

B

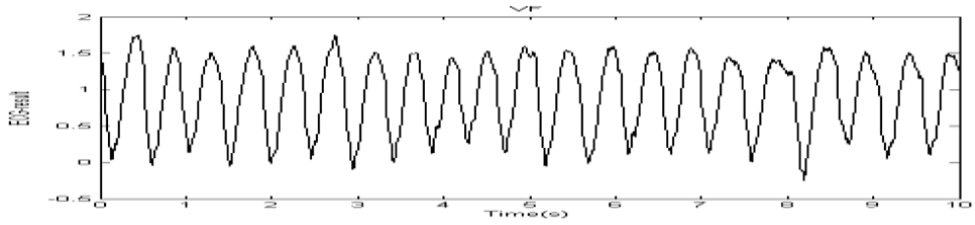

C

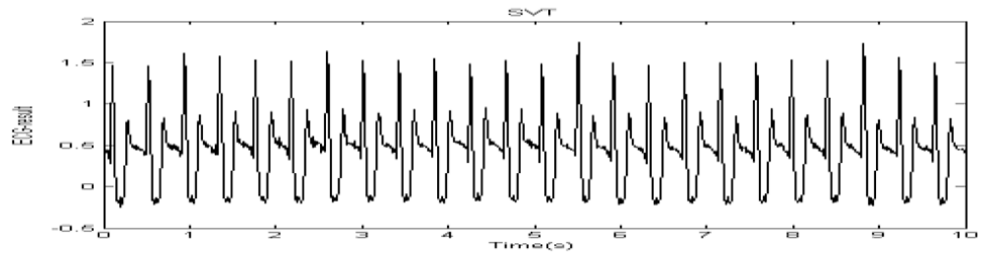

D

Figure 1: Instances of A) normal, B) Ventricular Tachycardia (VT), C) Ventricular Fibrillation (VF), D) Supraventricular Tachycardia (SVT) signals to demonstrate external similarities in the time domain. 
rithm is shown in Figure 2, consisting of five steps: 1) the preprocessing stage, where frequency, matching of the input ECG data and the application of time windows in order to extract identical time intervals from the data, is performed at this stage. 2) Conversion to the state space, 3 ) feature extraction from the developed data in the new space, 4) feature selection from the acquired data in the new space, and finally, 5) feature classification using the appropriate MLP algorithm.

\section{1) Preprocessing}

Due to extraction from different databases, data sampling rates have different values and in order to achieve a higher level of reliability, the data sampling rates were initially made identically in terms of value. Since the entire length of data is not required for the state space modeling and only a short-length signal from the original data is quite sufficient, only 8 seconds of the data signal length were considered for each patient's respective signal. A number of samples were obtained by applying an 8-second window over different parts of the data. Furthermore, it is worth noting that no overlapping exists between the applied windows to the data. The best performance can reach over an 8-second time interval [26].

2) Conversion to state space

Signal conversion to state space, as a useful and efficient method, was employed in order to expose more differences among the signals used in this study.

a) Theoretical Concept of State Space Reconstruction

It is rather necessary to find patterns not only in the time series itself but also in its conversion into a higher-dimensional space for each analyzed time series, such as electrocardiogram signals. The state space considered in this study is a special case of such a conversion. In fact, the space state is an n-dimensional space, in which a signal is plotted with respect to its delay. Each point in the state space is expressed by the following relation:

$$
\begin{gathered}
\bar{X}_{n}=\left[X_{n} X_{n-\tau} \ldots X_{n-(d-1) \tau}\right] ; \\
n=(1+(d-1)) \tau \ldots N
\end{gathered}
$$

Where $X_{n}$ is the $\mathrm{n}^{\text {th }}$ point in the time series, $\tau$ is the time delay, $\mathrm{d}$ is the time-space dimension, and $\mathrm{N}$ is the number of time series points. All the acquired points in the state space can be created using the following relation:

$$
X=\left[\begin{array}{c}
\bar{X}_{1+(d-1) \tau} \\
\bar{X}_{2+(d-1) \tau} \\
\vdots \\
\bar{X}_{N}
\end{array}\right]=\left[\begin{array}{ccc}
\bar{X}_{1+(d-1) \tau} & \cdots & \bar{X}_{1} \\
\vdots & \ddots & \vdots \\
\bar{X}_{N} & \cdots & \bar{X}_{N-(d-1) \tau}
\end{array}\right](2)
$$

According with (1) and (2) equations, the dimension should be initially determined prior to the time delay rate in order to create a state space. Different results are obtained from each of these values; thus, it is vital to determine the respective optimal values.

b) Time-delay algorithm

The time-delay algorithm reconstructs the signal in the state space. Moreover, according to the Takens's theorem [26], each signal can be considered as a state variable. In this study, the time-delay method was used to reconstruct the signal in the state space, where the signal is on the horizontal axis and the delay is on the vertical axis $[27,28]$. In other words, the

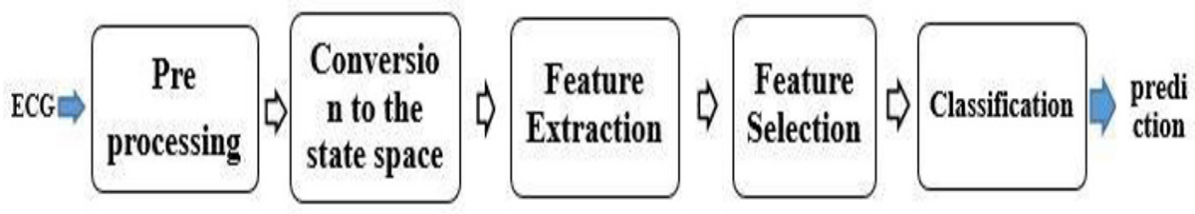

Figure 2: The block diagram representing the proposed algorithm. 
primary signal is considered as the state variable $X(t)$ and based on this method, the delayed form of the primary signal $X(t+\tau)$ is obtained, where $\tau$ is the signal delay. Plotting the two variables by points causes the initial signal to be mapped on the two-dimensional space. It should be noted that the signals were mapped on a two-dimensional space so that they can be used as an image [29]. The behavior of VT SVT, VF, and the normal signals is largely similar in the time domain; however, as shown in Figure 3, the differences between these signals were rather elevated in the state space.

Two common methods are used in order to estimate the most appropriate time delay, including the normalized mutual information (NMI) method and an experience-driven method. In the normalized mutual informa- tion method, the mutual information between the integer values of successive delay choices is determined, where the obtained result is used as an appropriate estimation of the optimal delay. In the second method, the delay is obtained experimentally and by conducting several classification tests [30]. In this study, the best value of $\tau$ in the variable $X(t+\tau)$ was determined using the normalized mutual information method [31], exceeding in the accuracy with respect to the mutual information method. This is achieved by first converting the signals used for classification to the state space and obtaining their image. Then, the NMI criterion estimates the level of similarity between the two images. Finally, this criterion is considered as a cost function in an optimization algorithm i.e. particle swarm optimization [32], where the minimum NMI values can be
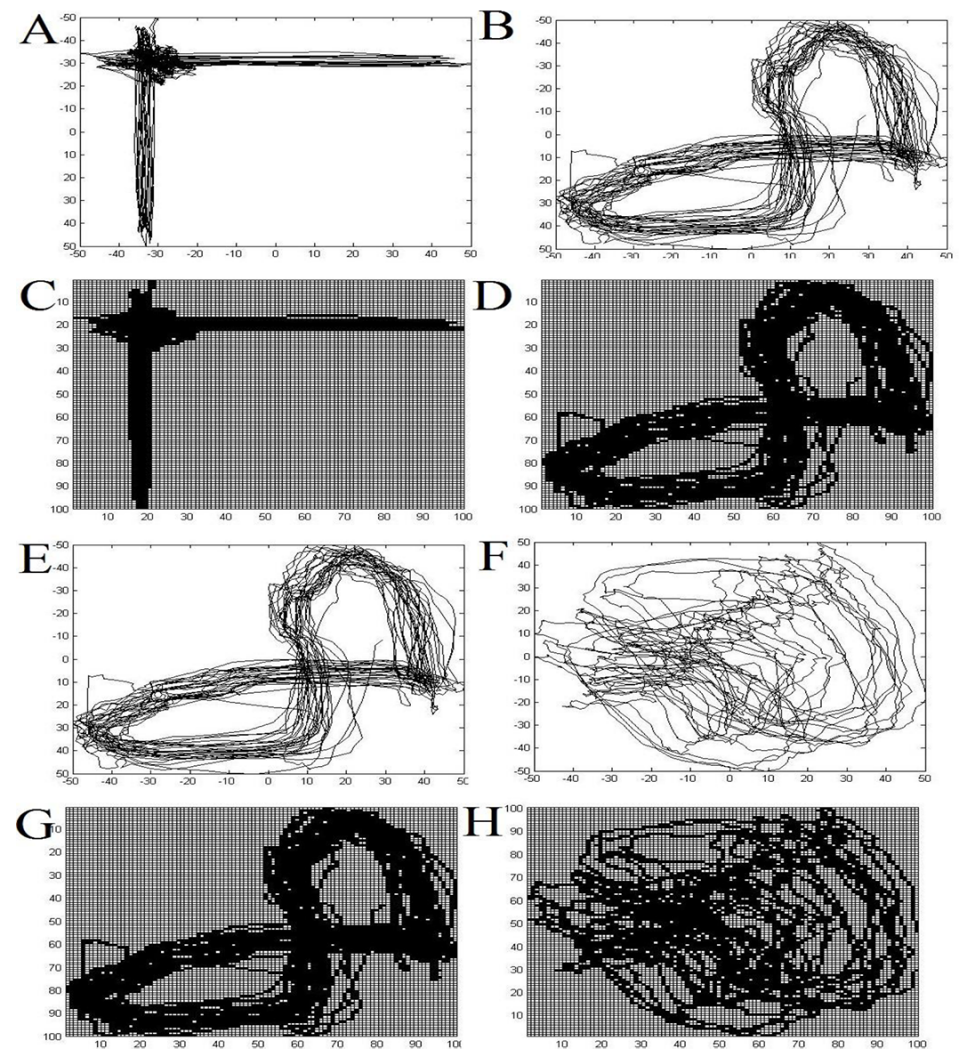

Figure 3: The representation for the signal transmitted to the state space. A) Normal signal B) Ventricular Tachycardia (VT) signal C) the gridded state space image for the normalized signal D) the gridded state space image for the VT E) Supraventricular Tachycardia (SVT) signal F) Ventricular Fibrillation (VF) signal G) the gridded state space image for the SVT H) the gridded phase space image for the VF. 
obtained for different values of $\tau$. The obtained value is an indication of the delay generating the maximum difference between the two images. The optimization algorithm was employed in order to facilitate the search process of the delay parameter. In addition, a variety of optimization methods exists, including PSO, Gradient Descent, Powell's algorithm, SA algorithm, Levenberg Marquardt algorithm, and Genetic algorithm. PSO algorithm, used in this study, and possesses several advantages compared to other algorithms, such as performing faster and an ability to adjust the accuracy [31]. Since the level of algorithm complexity is important in this section, the PSO algorithm was utilized, providing more accurate results than the other algorithms. Then, the respective appropriate delays for each test were individually estimated and extracted.

\section{3) Feature extraction}

SVT, VF, VT, and normal signals were mapped to a 100 by 100 image in the state space in order to further enhance the differences and create a comparison environment for them. Each empty box presents a pixel, where either value of 0 or 1 can be assigned to them. Moreover, image-processing techniques such as perimeter, area and the center of gravity detection and morphological operators can be used to extract the features and determine the data for artificial neural network training. In addition, while using the available concepts in the literature [23], it is rather possible to eliminate a large amount of common information from the images by applying a number of masks, which their efficacy can be proved empirically, and utilizing them for classification. In this regard, it should be noted that the masks and images dimension must be identical, where the total number of black pixels is the desired value after applying the masks. Additionally, a combination of mask and image processing techniques can be used in order to create a feature and, consequently, a higher quality classification. On the other hand, another group of features used in this study was the Gray-Level Co-Occurrence Matrix (GLCM) features [33]. Furthermore, unlike gray-level histograms, which are only capable of displaying spatial distribution and gray levels of the images, this group of features can simultaneously display information regarding the relative position and angular relation of the two pixels of the corresponding image. These features can be considered as one of the most widely used texture features, containing second-order statistical information of an image. In this study, seven main features associated with GLCM of images were used, including dissimilarity, contrast, correlation, variance, homogeneity, entropy, and energy, which are among the most optimal features of GLC.

Out of the mentioned criteria, the gravity center of images, internal area, and GLCM were of two output parameters representing the two co-ordinate axes. Out of the 8 masks shown in Figure 4, six masks Figure 4(A, C, D, E, F and $\mathrm{G})$ possessed higher levels of efficiency and were used in this research. Moreover, other criteria used for image differentiation were the application of two other masks, Figure 4 (B and $\mathrm{H}$ ), and their center of gravity, where the vertical and horizontal axis was considered for the first and second criteria, respectively. In overall, 10 features were acquired from these criteria. The occupied area of the images was regarded as the $11^{\text {th }}$ feature. After applying the opening morphological operator, the internal area was confirmed as the $12^{\text {th }}$ feature; thus, 12 features were extracted for the purpose of network training. In addition, 7 more GLCM features were extracted, evaluating the images in terms of texture. Finally, each of the introduced features was assessed and used to classify ECG signals by applying them as an MLP neural network classifier.

4) Feature selection

The performance and efficiency of a supervised learning algorithm can be strongly influenced by initial inputs. The purpose of feature selection techniques is to achieve the best subset compared to all of the extracted 

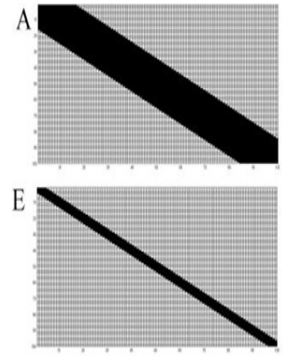
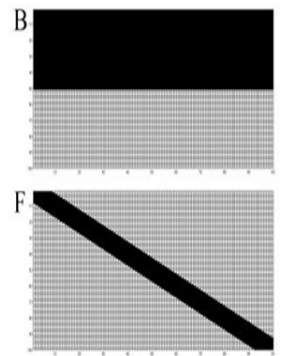
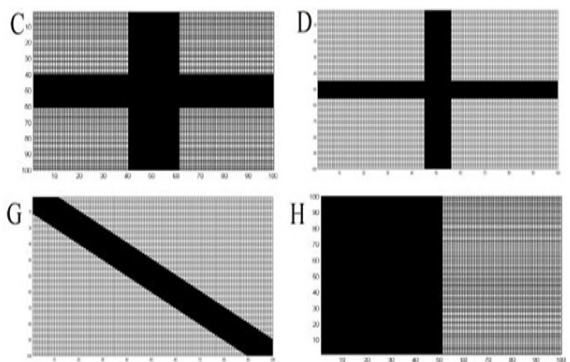

Figure 4: The masks utilized to remove common information, $(A),(E),(F)$ and $(G)$ with -45 degree angles and thicknesses of 15, 3, 7, and 10 pixels, (C) and (D) with thicknesses of 40 and 20 pixels, $(\mathrm{H})$ and $(\mathrm{B})$, which are vertical and horizontal, respectively, with thicknesses equivalent to 50 pixels.

feature in order to describe the inputs. In this paper, the Receiver Operating Characteristic (ROC) curve was used between classes in order to evaluate the efficiency of each feature in different classifications. Furthermore, more desirable features were extracted and utilized in the classification algorithm.

5) Classification

In this study, the multilayer perceptron (MLP) neural network was employed for the classification of previously obtained data [34]. Moreover, the activation sigmoid function in each layer and the Levenberg Marquardt algorithm, as an error backpropagation algorithm, for neural network training were implemented in MLP. In total, $70 \%$ of the data were utilized for training, $15 \%$ for validation, and $15 \%$ for the neural network testing. The data were selected randomly and the network efficiency was calculated by the Mean Square Error (MSE) method. In this network, the prescribed values for the performance and validation checks, as a mean to stop the algorithm, were considered at 100e-7 and 12 errors, respectively. The maximum number of iteration was also determined at 100 .

\section{Results}

In Tables 1 and 2, the experiment were carried out for each specified group, and the mean square error, accuracy, precision, sensitivity, F-measure parameter, and the average num- ber of errors were separately determined for each of the 12 extracted features. Essentially, the F-measure parameter is a criterion determining the resolution level in two of the data classes. Moreover, information regarding the F-measure parameter can be used in order to select a feature. This is carried out to examine the efficiency of the extracted features and to select the appropriate features for the final classification. The specified features in Table 2 are as follows:

1. Applying mask (g) to the image and counting the number of black pixels.

2. Applying mask (f) to the image and counting the number of black pixels.

3. Applying mask (e) to the image and counting the number of black pixels.

4. Applying mask (a) to the image and counting the number of black pixels.

Table 1: The required delay for converting each group to state space, as well as the associated normalized mutual information (NMI) ratio.

\begin{tabular}{ccc} 
Group & Delay(s) & NMI \\
\hline Normal-VF & 0.22 & -3.563 \\
\hline Normal-SVT & 0.19 & -4.47 \\
\hline VT-SVT & 0.22 & -2.746 \\
\hline VT-VF & 0.21 & -2.152
\end{tabular}

NMI: Normalized mutual information, VF: Ventricular Fibrillation, SVT: Supraventricular Tachycardia, VT: Ventricular Tachycardia 
5. Applying mask (c) to the image and counting the number of black pixels.

6 . Total internal area of the image and counting the number of black pixels.

7. Horizontal axis associated with the center of gravity of the image.

8. Vertical axis associated with the center of gravity of the image.

9. Applying mask (d) to the image and count- ing the number of black pixels.

10. Applying mask (b) to the image and the horizontal axis associated with the center of gravity of the image, obtained by applying the mask.

11. Applying mask (I) to the image and the vertical axis associated with the center of gravity of the image, obtained by applying the mask.

Table 2: Results obtained using the multilayer perceptron (MLP) neural network. The extracted features are separately fed to the neural network as inputs, and each result is obtained after 10 trials.

\begin{tabular}{|c|c|c|c|c|c|c|c|}
\hline Group & Feature & $\begin{array}{c}\text { Perform } \\
\text { (mse) }\end{array}$ & $\begin{array}{c}\text { Precision } \\
(\%)\end{array}$ & $\begin{array}{c}\text { Sensitivity } \\
(\%)\end{array}$ & $\begin{array}{c}\text { Accuracy } \\
(\%)\end{array}$ & $\begin{array}{c}\text { F-measure } \\
(\%)\end{array}$ & $\begin{array}{c}\text { Mean-Fal- } \\
\text { Number }\end{array}$ \\
\hline \multirow{12}{*}{$N-V F$} & 1 & 0.027 & 95.8 & 98.3 & 96.5 & 97 & 2.9 \\
\hline & 2 & 0.03 & 96.1 & 97.3 & 96.1 & 96.7 & 3.2 \\
\hline & 3 & 0.033 & 96 & 97.7 & 96.2 & 96.8 & 3.1 \\
\hline & 4 & 0.035 & 95.7 & 97.1 & 95.8 & 96.4 & 3.5 \\
\hline & 5 & 0.066 & 93.5 & 90.9 & 90.9 & 92 & 7.5 \\
\hline & 6 & 0.027 & 98.5 & 96.1 & 96.8 & 97.3 & 2.6 \\
\hline & 7 & 0.098 & 93.3 & 83.6 & 87.2 & 87.9 & 11.3 \\
\hline & 8 & 0.100 & 97.4 & 76.9 & 85.1 & 85.9 & 12.3 \\
\hline & 9 & 0.078 & 96.3 & 88.2 & 91 & 94.3 & 7.4 \\
\hline & 10 & 0.198 & 74 & 87 & 73.9 & 79 & 21.6 \\
\hline & 11 & 0.209 & 96.5 & 50.5 & 69.5 & 65.9 & 25.3 \\
\hline & 12 & 0.098 & 92.10 & 86.1 & 86.81 & 89 & 10.8 \\
\hline \multirow{12}{*}{ VT - SVT } & 1 & $5 e-09$ & 100 & 100 & 100 & 100 & 0 \\
\hline & 2 & $3.1 \mathrm{e}-09$ & 100 & 100 & 100 & 100 & 0 \\
\hline & 3 & $1.3 e-09$ & 100 & 100 & 100 & 100 & 0 \\
\hline & 4 & $1.3 e-09$ & 100 & 100 & 100 & 100 & 0 \\
\hline & 5 & 0.047 & 97.6 & 90.2 & 94.1 & 93.7 & 4 \\
\hline & 6 & 0.208 & 60.3 & 93.5 & 67 & 73.3 & 22.3 \\
\hline & 7 & 0.002 & 100 & 99.4 & 99.7 & 99.6 & 0.2 \\
\hline & 8 & 0.014 & 97.3 & 98.5 & 97.9 & 97.9 & 1.4 \\
\hline & 9 & 0.041 & 97 & 92.7 & 95 & 94.7 & 3.4 \\
\hline & 10 & $6.2 \mathrm{e}-09$ & 100 & 100 & 100 & 100 & 0 \\
\hline & 11 & 0.165 & 65.2 & 93.3 & 72.6 & 76.6 & 18.6 \\
\hline & 12 & 0.113 & 90.8 & 79.6 & 86.2 & 83.2 & 9.4 \\
\hline
\end{tabular}


Soheil Pashoutan, Shahriar Baradaran Shokouhi

\begin{tabular}{|c|c|c|c|c|c|c|c|}
\hline Group & Feature & $\begin{array}{l}\text { Perform } \\
\text { (mse) }\end{array}$ & $\begin{array}{c}\text { Precision } \\
(\%)\end{array}$ & $\begin{array}{c}\text { Sensitivity } \\
(\%)\end{array}$ & $\begin{array}{c}\text { Accuracy } \\
(\%)\end{array}$ & $\begin{array}{c}\text { F-measure } \\
(\%)\end{array}$ & $\begin{array}{c}\text { Mean-Fal- } \\
\text { Number }\end{array}$ \\
\hline \multirow{12}{*}{$\begin{array}{l}\text { Normal } \\
\text { - SVT }\end{array}$} & 1 & 0.055 & 93.8 & 93.4 & 92.6 & 93.6 & 6.2 \\
\hline & 2 & 0.063 & 92.7 & 93.2 & 91.9 & 92.9 & 6.8 \\
\hline & 3 & 0.062 & 92.1 & 93 & 91.3 & 92.5 & 7.3 \\
\hline & 4 & 0.068 & 92.3 & 93 & 91.4 & 92.6 & 7.2 \\
\hline & 5 & 0.11 & 87.1 & 90 & 86.3 & 88.5 & 11.4 \\
\hline & 6 & 0.108 & 84.1 & 93.6 & 85.8 & 88.5 & 11.9 \\
\hline & 7 & 0.180 & 81.6 & 73.9 & 75 & 77.4 & 20.1 \\
\hline & 8 & 0.245 & 58.3 & 100 & 58.3 & 73.65 & 35 \\
\hline & 9 & 0.115 & 89.5 & 83.9 & 84.6 & 86.2 & 12.5 \\
\hline & 10 & 0.231 & 69.68 & 70.4 & 67.7 & 69.5 & 29.6 \\
\hline & 11 & 0.192 & 81.92 & 60.2 & 63.7 & 64.4 & 30.5 \\
\hline & 12 & 0.103 & 85 & 93 & 86.7 & 88.4 & 11.1 \\
\hline \multirow{12}{*}{ VF - VT } & 1 & 0.013 & 100 & 95.8 & 97.9 & 98 & 1.4 \\
\hline & 2 & 0.021 & 100 & 97 & 98.5 & 98.47 & 1 \\
\hline & 3 & 0.012 & 100 & 97 & 98.5 & 98.47 & 1 \\
\hline & 4 & 0.012 & 100 & 97 & 98.5 & 98.47 & 1 \\
\hline & 5 & 0.55 & 100 & 87.6 & 94.1 & 93.39 & 4 \\
\hline & 6 & 0.2 & 60.6 & 89.7 & 66.4 & 72.2 & 20.6 \\
\hline & 7 & 0.111 & 96 & 73.2 & 85.1 & 82.9 & 10.3 \\
\hline & 8 & 0.120 & 97.9 & 72.5 & 85 & 83.1 & 10.3 \\
\hline & 9 & 0.056 & 100 & 90.9 & 95.6 & 95.23 & 3 \\
\hline & 10 & 0.007 & 100 & 99.4 & 99.7 & 99.5 & 0.2 \\
\hline & 11 & 0.151 & 76.5 & 83 & 79 & 79.5 & 14.4 \\
\hline & 12 & 0.268 & 53.3 & 50.6 & 53.8 & 50 & 31.7 \\
\hline
\end{tabular}

VF: Ventricular Fibrillation, VT: Ventricular Tachycardia, SVT: Supraventricular Tachycardia

12. Applying the opening operator, calculation of the obtained image area, and counting the number of black pixels.

13-19. GLCM feature.

The results in Table 2 were applied to the neural network, as input, after separately applying each feature in the respective group. It should be noted that the MLP neural network was trained with one neuron in the hidden layer and one in the output layer. Furthermore, the average results were recorded after 10 it- erations for a higher level of credibility. In the next step, the appropriate features providing us with a greater level of resolution were selected in each of the groups and used in a new MLP neural network classifier as input data. In addition, the results were compared to a combination of all the features. In this section, the MLP neural network possesses two neurons in the hidden layer. Table 3 demonstrates the results of this experiment.

In the final stage, the groups were divided 
into four classes of normal-VF-VT, normalVF-SVT, VT-VF-SVT and four classes of normal-VT-VF-SVT. Moreover, appropriate features were used for the MLP artificial neural network training with 5 neurons in the hidden layer. The obtained results from this stage are presented in Table 4.

Over the past few years, numerous stud- ies have been carried out in order to classify the ECG signals, however, few of them have investigated a method that has the ability to simultaneously classify three signals of VF, VT, SVT, and the normal signal. The results of several related studies, along with their classification groups and the extracted features, are presented in Table 5.

Table 3: Results obtained using the multilayer perceptron (MLP) neural network. The features employed as the network inputs are globally optimal features and most distinctive ones, represented in Table 2.

\begin{tabular}{|c|c|c|c|c|c|c|c|}
\hline Group & Feature & $\begin{array}{c}\text { Perform } \\
\text { (mse) }\end{array}$ & $\begin{array}{c}\text { Precision } \\
(\%) \\
\end{array}$ & $\begin{array}{c}\text { Sensitivity } \\
(\%)\end{array}$ & $\begin{array}{c}\text { Accuracy } \\
(\%)\end{array}$ & $\begin{array}{c}\text { F-Measure } \\
(\%)\end{array}$ & Mean-Fal-Number \\
\hline \multirow{3}{*}{$\mathbf{N}-\mathbf{V F}$} & $1,6,9$ & 0.004 & 99.2 & 100 & 99.5 & 99.5 & 0.4 \\
\hline & 1,6 & 0.008 & 99.2 & 100 & 99.5 & 99.5 & 0.4 \\
\hline & $13-19$ & 0.008 & 99.63 & 99.63 & 99.65 & 99.63 & 0.6 \\
\hline \multirow{5}{*}{ VT - SVT } & 7,8 & $6.2 \mathrm{e}-09$ & 100 & 100 & 100 & 100 & 0 \\
\hline & 1,9 & $1.04 \mathrm{e}-08$ & 100 & 100 & 100 & 100 & 0 \\
\hline & 10,12 & $4.6 \mathrm{e}-06$ & 100 & 100 & 100 & 100 & 0 \\
\hline & 10,11 & $1.2 \mathrm{e}-08$ & 100 & 100 & 100 & 100 & 0 \\
\hline & $13-19$ & $5.5 \mathrm{e}-08$ & 100 & 100 & 100 & 100 & 0 \\
\hline \multirow{2}{*}{ N - SVT } & $1,2,3$ & 0.049 & 94.1 & 93.9 & 93 & 93.7 & 5.8 \\
\hline & $13-19$ & 0.025 & 97.08 & 87.16 & 94.98 & 94.73 & 3.9 \\
\hline \multirow{3}{*}{ VF - VT } & $1,2,3$ & 0.004 & 100 & 98.8 & 99.4 & 99.3 & 0.2 \\
\hline & 3,10 & 0.001 & 100 & 99.4 & 99.7 & 99.7 & 0.2 \\
\hline & $13-19$ & $8.2 \mathrm{e}-08$ & 100 & 100 & 100 & 100 & 0 \\
\hline VT-N & $13-19$ & $4.26 \mathrm{e}-08$ & 100 & 100 & 100 & 100 & 0 \\
\hline
\end{tabular}

VF: Ventricular Fibrillation, VT: Ventricular Tachycardia, SVT: Supraventricular Tachycardia

Table 4: Results obtained using the multilayer perceptron (MLP) neural network. The features employed as the network inputs are globally optimal features and most distinctive ones, represented in this table, groups with 3 and 4 classes are classified.

\begin{tabular}{cccccccc} 
Group & \multicolumn{2}{c}{$\mathbf{V T}-\mathbf{N}-\mathbf{V F}$} & \multicolumn{2}{c}{$\mathbf{N}-\mathbf{S V T}-\mathbf{V F}$} & VT-VF-SVT & \multicolumn{2}{c}{ VT-SVT-VF-N } \\
\hline Feature & $1-6-10$ & $1-6-10-12$ & $1-6-12$ & $1-6-9-12$ & $13-19$ & $1-6-9-12$ & $13-19$ \\
\hline Perform (mse) & 0.0003 & $1.9 \mathrm{e}-09$ & 0.030 & 0.023 & 0.012 & 0.025 & 0.036 \\
\hline Accuracy (\%) & 99.4 & 100 & 94.8 & 96.3 & 96.5 & 95 & 86.57 \\
\hline Mean-Fal-Number & 0.7 & 0 & 5.3 & 4.1 & 2.3 & 7.5 & 8.8
\end{tabular}

VT: Ventricular Tachycardia, VF: Ventricular Fibrillation, SVT: Supraventricular Tachycardia 
Table 5: Comparing the results obtained using the proposed system with other related resources.

\begin{tabular}{|c|c|c|c|c|c|}
\hline Study & Feature & Precision & Sensitivity & Accuracy & Group \\
\hline Ruiz [35] & Cross Validation & - & 96.7 & - & $\mathrm{N}-\mathrm{VT}$ \\
\hline Riasi [36] & Complex network theory & 88.8 & 85 & - & N-VT \\
\hline Riasi [2] & Morphology & 100 & 88 & - & $\mathrm{N}-\mathrm{VT}$ \\
\hline Roopai [29] & $\begin{array}{l}\text { Difference Method in state } \\
\text { space }\end{array}$ & - & - & 91.51 & N-VT \\
\hline This study & The proposed method & 100 & 100 & 100 & N-VT \\
\hline Choi-williams [23] & $\begin{array}{c}\text { Short time Fourier Trans- } \\
\text { form }\end{array}$ & - & - & 90 & VT-VF \\
\hline Sarvestani [23] & Mask in state space & - & - & 100 & VT-VF \\
\hline Roopai [29] & $\begin{array}{l}\text { Difference Method in state } \\
\text { space }\end{array}$ & - & - & 89.8 & VT-VF \\
\hline This study & The proposed method & 100 & 100 & 100 & VT-VF \\
\hline Ruiz [35] & Cross Validation & - & 90.2 & - & VF-N \\
\hline Amann [22] & $\begin{array}{c}\text { Number of pixel in state } \\
\text { space }\end{array}$ & 97.8 & 79 & 96.2 & VF-N \\
\hline Roopai [29] & Similary method & - & - & 94.13 & VF-N \\
\hline This study & The proposed method & 99.2 & 100 & 99.5 & VF-N \\
\hline
\end{tabular}

VT: Ventricular Tachycardia, VF: Ventricular Fibrillation

\section{Discussion}

In this study, the data were divided into nine groups of normal-VF, VT-SVT, normal-SVT, VT-VF, normal-VT, normal-VF-VT, normal- SVT-VF, VT-VF-SVT and normal-VTVF-SVT in order to evaluate the efficiency of the proposed algorithm. This partitioning was performed based on the level of features correlation; finally, features were individually extracted and classified for the data of each group. Moreover, the efficiency of the proposed system was achieved by evaluating the results from each group. In this research, the obtained results are divided into three general sections. In the first section, the optimal values of time delay $\tau$, which are required for each of the mentioned groups, are separately obtained in accordance with the method described in Section 4. As previously stated, the delay is obtained using the PSO algorithm and the cost function associated with the normalized mutual information. In addition, in order to increase the validity of the obtained values, 10 samples were selected in each group, where the experiment was repeated for each of these samples. Then, the specific value reported was repeated more than others in these 10 experiments, recognized as the optimum and final value of time delay. The delay search field was from 0 to 4 seconds and after 20 iterations, 20 final optimal data were extracted in each experiment. Then, the most optimal value obtained from the entire 10 experiment was considered as the value of delay. It is worth noting that the final values of time delay may not be the most optimal in a number of cases, however, it is certainly one of the best time delay values, including the maximum of the 
data in the respective group. Additionally, although delay time values of less than 0.05 seconds are considered as optimal values in the NMI algorithm, their results are not perceived as desirable. Thus, the values within this interval should be neglected. The most efficient delay is obtained empirically from the optimal values for each group and the optimal time delay is constant in all of the experiments for each group, as shown in Table 1. Figure 5 also illustrates the variation of normalized mutual information with respect to delay changes along with the time delay value in each group. Subsequently, the signal is transmitted to the state space while taking the appropriate delay into account, where the result is plotted in a two-dimensional space and used as an image.

In the second section, a total number of 19 features were extracted from the obtained image for the artificial neural network training. Out of these 19 features, two features represented the length and width of the center of gravity. One feature represented the total area of the image and six features were obtained by applying the efficient, as shown in Figure 4 masks, for the image and counting the number of black pixels. Two other features were obtained by applying the $\mathrm{H}$ and $\mathrm{B}$ masks, shown in Figure 4, and then taking the length and width of the center of gravity into account, respectively. Furthermore, the $12^{\text {th }}$ feature was also obtained by applying the opening morphological operator and then determining the total area. Finally, seven GLCM features were extracted from the images. Therefore, all of the 19 features were extracted and laid the groundwork for the artificial neural network training. Figure 6 depicts a number of the obtained features after applying the mask.

In the last section, the obtained features were initially applied to the appropriate MLP neural network, according to the performed classification.

\section{Conclusion}

In this paper, a new approach was developed to classify the abnormal signals obtained from an ECG such as VT, VF, and SVT compared to a normal signal. In the first step, these signals were transmitted to the state space in order to obtain valuable information from each of them. Finally, the corresponding signals were mapped to a 100 by 100 image. The parameter $\tau$ was considered as the delay and one of the main challenges in this research was obtaining an optimal value of $\tau$. Initially, data were divided into nine groups of normal-VF, VT-SVT, normal-SVT, VT-VF, normal-VT, normal-VF-VT, normal-SVT-VF, VT-VFSVT and normal-VT-VF-SVT. Then, using the NMI algorithm, the obtained level of similarity between the images was measured in terms of the variations of delay in the previously mentioned groups. Finally, the optimal value was obtained separately in each group using the PSO optimization algorithm. Therefore, the most optimal time delay can be employed in each group for the transmission to
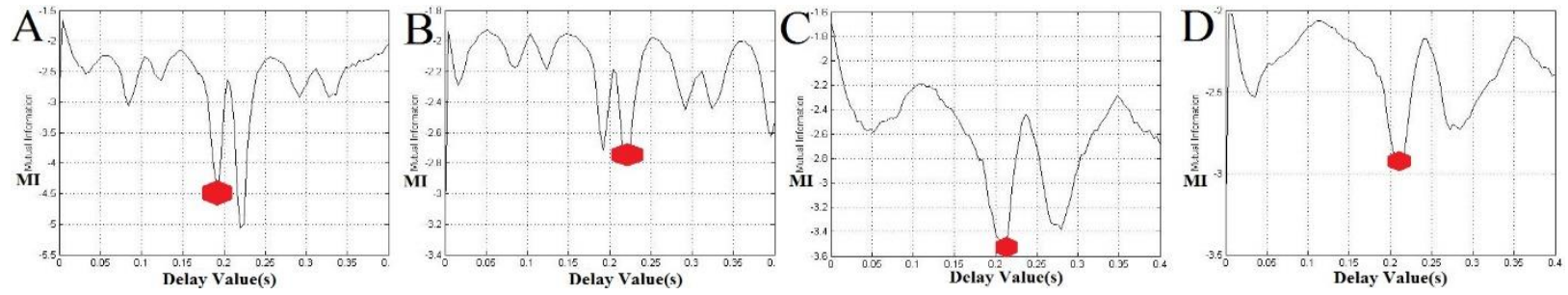

Figure 5: Time delay and normalized mutual information (NMI) ratios, along with the illustration of the globally optimal time delay for (A) Normal-Supraventricular Tachycardia (SVT), (B) Ventricular Tachycardia (VT)-SVT, (C) N-Ventricular Fibrillation (VF) and (D) VF-VT 

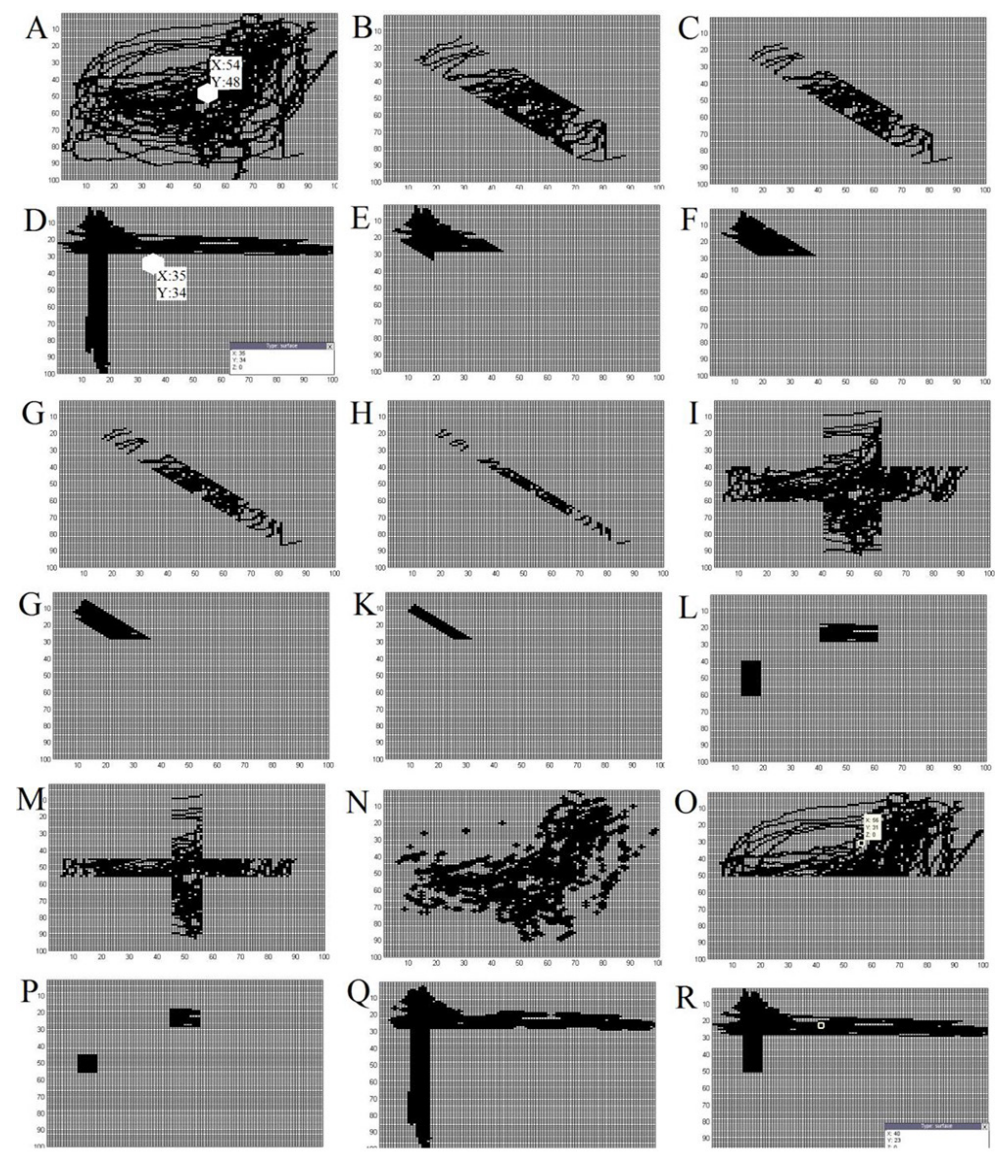

Figure 6: The results were obtained after applying the masks presented in Figure 4 and some of the introduced features. In these figures, rows 1,3 , and 5, refer to the VF images, while $2^{\text {nd }}, 4^{\text {th }}$, and $6^{\text {th }}$ rows represent the normal images. In addition, Figures A) and D) illustrate the center of mass; B) and E) demonstrate applying -45 degrees masks with thicknesses of 15 pixels; C) and F) illustrates applying -45 degrees masks with thicknesses of 10 pixels; $\mathrm{G}$ ) and J) demonstrate applying -45degrees masks with thicknesses of 7 pixels; $H$ ) and $K$ ) illustrate applying -45 degrees masks with thicknesses of 3 pixels images shown in I) and L); results from applying the mask presented in Figure $4(C), M)$ and P) from applying the mask presented in Figure 4 (D), and images $\mathrm{N}$ ) and Q) represent applying the "opening" operator, followed by considering the area, and finally $\mathrm{O}$ ) and R) images demonstrate applying the mask presented in Figure 4 (B) and determining the center of mass in the images.

state space. This value assists to minimize the probability of obtaining similar images. In the second section, a total number of 19 features were extracted from the image; then, all of the features were combined, where three and four classes were separately selected for each data group, according to the type of data in the group. The selected features exhibited a high level of performance in terms of data classification. Finally, each feature group was ap- plied to the MLP classifier and the respective accuracy was determined. The most optimal structure of the classifier was also obtained empirically in a trial-and-error process.

The sixth feature in the N-VF group, the first, second, third, fourth and $10^{\text {th }}$ features in the VT-SVT group, the first feature in the N-SVT group, and the $10^{\text {th }}$ feature in the VT-VF group were considered as the most appropriate features. In the next section, a number of features 
Classification of ECG Signals

were used in the neural network training and testing. The results of classification accuracy were determined at $99.5 \%, 100 \%, 94.98 \%$, $100 \%$ and $100 \%$, respectively, compared to the related studies, and reported higher levels of efficiency. A more complex neural network system was the only disadvantage of a multifeature rather than a single feature system. In the last section, groups with three and four classes are presented and the desirable neural network was trained using the appropriate features. Moreover, the data were tested in this network. The accurate results were determined $100 \%$ for the VT-VF-normal group, $96.3 \%$ for the normal-VF-SVT group, $96.5 \%$ for the VTVF-SVT and 95\% for the VT-VF-SVT-normal group. In addition, other novelties of this paper include the comprehensiveness of the proposed system in the classification of other data groups. Other related studies mainly focus on the classification of VT and VF signals. Compared to these studies, our proposed system significantly performed better. However, the main contribution of this research was in the separation of normal, VT, and VF signals and the normal-VF-SVT signal, VT-VF-SVT as well as all of the four normal, VT, VF, and SVT signals. Thus, the accurate values were determined at $100 \%, 99.5 \%, 96.5 \%$ and $95 \%$ of the proposed system, respectively

\section{Conflict of Interest}

None

\section{References}

1. Theodoridis S, Koutroumbas K. Pattern recognition 2003. Elsevier; 2009.

2. Riasi A, Mohebbi M, editors. Prediction of ventricular tachycardia using morphological features of ECG signal. The International Symposium on Artificial Intelligence and Signal Processing (AISP); IEEE; 2015. p. 170-5. doi: 10.1109/ aisp.2015.7123515.

3. Kelly BB, Fuster V. Promoting cardiovascular health in the developing world: a critical challenge to achieve global health. Washington: National Academies Press; 2010.

4. Dantas AP, Jimenez-Altayo F, Vila E. Vascular ag- ing: facts and factors. Front Physiol. 2012;3:325. doi: 10.3389/fphys.2012.00325. PubMed PMID: 22934073. PubMed PMCID: PMC3429093.

5. Goldschlager N, Goldman M. Effects of drugs and electrolytes on the electrocardiogram. Principles of Clinical Electrocardiography Appleton and Lange, East Norwalk. 1989:256-71.

6. Kolettis TM. Coronary artery disease and ventricular tachyarrhythmia: pathophysiology and treatment. Curr Opin Pharmacol. 2013;13:210-7. doi: 10.1016/j.coph.2013.01.001. PubMed PMID: 23357129.

7. Joo S, Huh S-J, Choi K-J, editors. A predictor for ventricular tachycardia based on heart rate variability analysis. IEEE Biomedical Circuits and Systems Conference (BioCAS); San Diego, CA, USA: IEEE; 2011. p. 409-411. doi: 10.1109/BioCAS.2011.6107814.

8. Li Q, Rajagopalan C, Clifford GD. Ventricular fibrillation and tachycardia classification using a machine learning approach. IEEE Trans Biomed Eng. 2014;61:1607-13. doi: 10.1109/ TBME.2013.2275000. PubMed PMID: 23899591.

9. Li Y, Pang Y, Wang J, Li X. Patient-specific ECG classification by deeper CNN from generic to dedicated. Neurocomputing. 2018;314:336-46. doi: 10.1016/j.neucom.2018.06.068.

10. Thakor NV, Zhu YS, Pan KY. Ventricular tachycardia and fibrillation detection by a sequential hypothesis testing algorithm. IEEE Trans Biomed Eng. 1990;37:837-43. doi: 10.1109/10.58594. PubMed PMID: 2227970.

11. Chen S, Thakor NV, Mower MM. Ventricular fibrillation detection by a regression test on the autocorrelation function. Med Biol Eng Comput. 1987;25:241-9. doi: 10.1007/bf02447420. PubMed PMID: 3329694.

12. Zhang XS, Zhu YS, Thakor NV, Wang ZZ. Detecting ventricular tachycardia and fibrillation by complexity measure. IEEE Trans Biomed Eng. 1999;46:54855. doi: 10.1109/10.759055. PubMed PMID: 10230133.

13. Barro S, Ruiz R, Cabello D, Mira J. Algorithmic sequential decision-making in the frequency domain for life threatening ventricular arrhythmias and imitative artefacts: a diagnostic system. J Biomed Eng. 1989;11:320-8. doi: 10.1016/01415425(89)90067-8. PubMed PMID: 2755113.

14. Addison PS, Watson JN, Clegg GR, Holzer M, Sterz $F$, Robertson CE. Evaluating arrhythmias in ECG signals using wavelet transforms. IEEE Eng Med Biol Mag. 2000;19:104-9. doi: 10.1109/51.870237. 
PubMed PMID: 11016036.

15. Alonso-Atienza F, Morgado E, Fernandez-Martinez L, Garcia-Alberola A, Rojo-Alvarez JL. Detection of life-threatening arrhythmias using feature selection and support vector machines. IEEE Trans Biomed Eng. 2014;61:832-40. doi: 10.1109/ TBME.2013.2290800. PubMed PMID: 24239968.

16. Zhou SH, Rautaharju PM, Calhoun HP, editors. Selection of a reduced set of parameters for classification of ventricular conduction defects by cluster analysis. Proceedings of Computers in Cardiology Conference; London, UK: IEEE; 1993. p. 879-82. doi: 10.1109/CIC.1993.378298.

17. Afonso VX, Tompkins WJ. Detecting ventricular fibrillation. IEEE Eng Med Biol Mag. 1995;14:152-9. doi: 10.1109/51.376752 .

18. Ham FM, Han S. Classification of cardiac arrhythmias using fuzzy ARTMAP. IEEE Trans Biomed Eng. 1996;43:425-30. doi: 10.1109/10.486263. PubMed PMID: 8626192.

19. Finelli CJ. The time-sequenced adaptive filter for analysis of cardiac arrhythmias in intraventricular electrograms. IEEE Trans Biomed Eng. 1996;43:811-9. doi: 10.1109/10.508543. PubMed PMID: 9216153.

20. Golrizkhatami Z, Acan A. ECG classification using three-level fusion of different feature descriptors. Expert Systems with Applications. 2018;114:5464. doi: 10.1016/j.eswa.2018.07.030.

21. Dong X, Wang C, Si W. ECG beat classification via deterministic learning. Neurocomputing. 2017;240:112. doi: 10.1016/j.neucom.2017.02.056.

22. Amann A, Tratnig R, Unterkofler K. Detecting ventricular fibrillation by time-delay methods. IEEE Trans Biomed Eng. 2007;54:174-7. doi: 10.1109/ TBME.2006.880909. PubMed PMID: 17260872.

23. Sarvestani RR, Boostani R, Roopaei M. VT and VF classification using trajectory analysis. Nonlinear Analysis: Theory, Methods \& Applications. 2009;71:e55-e61. doi: 10.1016/j.na.2008.10.015.

24. Lloyd MA, Murphy JG. Mayo Clinic Cardiology: Board Review Questions and Answers. CRC Press; 2007. doi: 10.1201/b14443.

25. Goldberger AL, Amaral LA, Glass L, Hausdorff JM, Ivanov PC, Mark RG, et al. PhysioBank, PhysioToolkit, and PhysioNet: components of a new research resource for complex physiologic signals. Circulation. 2000;101(23):E215-20. PubMed PMID: 10851218.

26. Cao L. Practical method for determining the minimum embedding dimension of a scalar time series. Physica D: Nonlinear Phenomena. 1997;110:43-
50. doi: 10.1016/s0167-2789(97)00118-8.

27. Rafal R, Pawel L, Krzysztof K, Bogdan K, Jerzy W. Chatter identification methods on the basis of time series measured during titanium superalloy milling. International Journal of Mechanical Sciences. 2015;99:196-207. doi: 10.1016/j.ijmecsci.2015.05.013.

28. Rusinek R, Weremczuk A, Kecik K, Warminski J. Dynamics of a time delayed Duffing oscillator. International Journal of Non-Linear Mechanics. 2014;65:98-106.

29. Roopaei M, Boostani R, Sarvestani RR, Taghavi MA, Azimifar Z. Chaotic based reconstructed phase space features for detecting ventricular fibrillation. Biomedical Signal Processing and Control. 2010;5:318-27. doi: 10.1016/j.bspc.2010.05.003.

30. Khan A, Rehman S, Abbas M, Ahmad A. On the mutual information of relaying protocols. Physical Communication. 2018;30:33-42. doi: 10.1016/j. phycom.2018.07.005.

31. Ayatollahi F, Shokouhi SB, Ayatollahi A. A new hybrid particle swarm optimization for multimodal brain image registration. Journal of Biomedical Science and Engineering. 2012;5:153. doi: 10.4236/ jbise.2012.54020.

32. Wachowiak MP, Smolíková R, Zheng Y, Zurada JM, Elmaghraby AS. An approach to multimodal biomedical image registration utilizing particle swarm optimization. IEEE Transactions on Evolutionary Computation. 2004;8:289-301. doi: 10.1109/ tevc.2004.826068.

33. Öztürk S, Akdemir B. Application of feature extraction and classification methods for histopathological image using GLCM, LBP, LBGLCM, GLRLM and SFTA. Proc Comput Sci. 2018;132:40-6. doi: 10.1016/j.procs.2018.05.057.

34. Tatar A, Naseri S, Bahadori M, Hezave AZ, Kashiwao $T$, Bahadori $A$, et al. Prediction of carbon dioxide solubility in ionic liquids using MLP and radial basis function (RBF) neural networks. Journal of the Taiwan Institute of Chemical Engineers. 2016;60:151-64. doi: 10.1016/j.jtice.2015.11.002.

35. Ruiz J, Aramendi E, De Gauna SR, Lazkano A, Leturiondo L, Gutierrez J, editors. Distinction of ventricular fibrillation and ventricular tachycardia using cross correlation. Computers in Cardiology; Greece: IEEE; 2003. doi: 10.1109/CIC.2003.1291259.

36. Li X, Dong Z. Detection and prediction of the onset of human ventricular fibrillation: an approach based on complex network theory. Physical Review E. 2011;84(6):062901. doi: 10.1103/PhysRevE.84.062901. PubMed PMID: 22304137. 